\title{
Comparison of the Effects of Various Antidiabetic Medication on Bone Mineral Density in Patients with Type 2 Diabetes Mellitus
}

\begin{abstract}
Jeonghoon $\mathrm{Ha}^{1}$, Yejee $\mathrm{Lim}^{2}$, Mee Kyoung Kim${ }^{3}$, Hyuk-Sang Kwon ${ }^{3}$, Ki-Ho Song ${ }^{3}$, Seung Hyun $\mathrm{Ko}^{4}$, Moo Il Kang ${ }^{1}$, Sung Dae Moon ${ }^{5}$, Ki-Hyun Baek ${ }^{3}$

${ }^{1}$ Division of Endocrinology and Metabolism, Department of Internal Medicine, Seoul St. Mary's Hospital, College of Medicine, The Catholic University of Korea, Seoul; ${ }^{2}$ Division of General Internal Medicine, Department of Internal Medicine, Seoul National University Bundang Hospital, Seongnam; ${ }^{3}$ Division of Endocrinology and Metabolism, Department of Internal Medicine, Yeouido St. Mary's Hospital, College of Medicine, The Catholic University of Korea, Seoul; ${ }^{4}$ Division of Endocrinology and Metabolism, Department of Internal Medicine, St. Vincent's Hospital, College of Medicine, The Catholic University of Korea, Suwon; ${ }^{5}$ Division of Endocrinology and Metabolism, Department of Internal Medicine, Incheon St. Mary's Hospital, College of Medicine, The Catholic University of Korea, Incheon, Korea
\end{abstract}

Background: Prospective comparative studies on the effects of various antidiabetic agents on bone metabolism are limited. This study aimed to assess changes in bone mass and biochemical bone markers in postmenopausal patients with type 2 diabetes mellitus (T2DM).

Methods: This prospective, multicenter, open-label, comparative trial included 264 patients with T2DM. Patients who had received a metformin, or sulfonylurea/metformin combination (Group 1); a thiazolidinedione combination (Group 2); a dipeptidyl peptidase-4 inhibitor (gemigliptin) combination (Group 3); or an sodium-glucose cotransporter 2 inhibitor (empagliflozin) combination (Group 4) were prospectively treated for 12 months; bone mineral density (BMD) and bone turnover marker (BTM) changes were evaluated. Results: The femoral neck BMD percentage changes were $-0.79 \% \pm 2.86 \%$ (Group 1), $-2.50 \% \pm 3.08 \%$ (Group 2), $-1.05 \% \pm 2.74 \%$ (Group 3), and $-1.24 \% \pm 2.91 \%$ (Group 4) $(P<0.05$ ). The total hip BMD percentage changes were $-0.57 \% \pm 1.79 \%$ (Group 1), $-1.74 \% \pm 1.48 \%$ (Group 2), $-0.75 \% \pm 1.87 \%$ (Group 3), and $-1.27 \% \pm 1.72 \%$ (Group 4) ( $P<0.05$ ). Mean serum BTM (C-terminal type 1 collagen telopeptide and procollagen type 1 amino-terminal propeptide) levels measured during the study period did not change over time or differ between groups.

Conclusion: Significant bone loss in the femoral neck and total hip was associated with thiazolidinedione combination regimens. However, bone loss was not significantly associated with combination regimens including gemigliptin or empagliflozin. Caution

Received: 10 March 2021, Revised: 6 May 2021, Accepted: 7 June 2021

Corresponding authors: Ki-Hyun Baek

Division of Endocrinology and Metabolism, Department of Internal Medicine, Yeouido St. Mary's Hospital, College of Medicine, The Catholic University of Korea, 10 63-ro, Yeongdeungpo-gu, Seoul 07345, Korea

Tel: +82-2-3779-1400, Fax: +82-2-708-3132, E-mail: drbkh@catholic.ac.kr

Sung Dae Moon

Division of Endocrinology and Metabolism, Department of Internal Medicine, Incheon St. Mary's Hospital, College of Medicine, The Catholic University of Korea, 56 Dongsu-ro, Bupyeong-gu, Incheon 21431, Korea

Tel: +82-32-280-5841, Fax: +82-32-280-5210, E-mail: sungdaem@gmail.com

\section{Copyright $@ 2021$ Korean Endocrine Society}

This is an Open Access article distributed under the terms of the Creative Commons Attribution Non-Commercial License (https://creativecommons.org/ licenses/by-nc/4.0/) which permits unrestricted non-commercial use, distribution, and reproduction in any medium, provided the original work is properly cited. 
should be exercised during treatment with antidiabetic medications that adversely affect the bone in patients with diabetes at a high risk of bone loss.

Keywords: Osteoporosis; Diabetes mellitus; Bone density; Thiazolidinediones; Sodium-glucose transporter 2 inhibitors

\section{INTRODUCTION}

Osteoporosis is defined as low bone mass and microarchitectural bone deterioration leading to increased bone fragility and fracture susceptibility [1]. Osteoporosis is a systemic skeletal disease that progresses gradually, and as the elderly population increases, the worldwide prevalence and associated socioeconomic burden are rising [2-4].

Bone is an important target organ subject to diabetic complications, and there is increasing evidence of a link between diabetes and osteoporosis. Type 1 diabetes is an important secondary cause of osteoporosis and is characterized by lower bone mass and higher fracture than those observed in the healthy population [5]. In patients with type 2 diabetes mellitus (T2DM), the bone mineral density (BMD) of the lumbar spine and hip was found to be normal or even higher than that of healthy subjects, and the results were consistent even after adjusting for age, sex, ethnicity, and body mass index [6,7]. However, the risk of fragility fractures is higher in patients with T2DM. An increased risk of fracture in patients with T2DM is associated with the duration of diabetes, the presence of diabetes complications, the type of antidiabetic medication, or frequent falls $[5,7,8]$. In the Women's Health Initiative Observational Study, women with T2DM were at increased risk for fractures despite BMD being increased; this result remained consistent even after adjustment for multiple risk factors for fracture [8].

Some antidiabetic medications are known to increase fractures in patients with diabetes. According to the Diabetes Outcome Progression Trial (ADOPT), fractures were more common in patients treated with a peroxisome proliferator-activated receptor $\gamma$ agonist than in the patients treated with metformin or glyburide [9]. Recent data from a randomized phase 3 study suggest that canagliflozin, a sodium-glucose cotransporter 2 inhibitor (SGLT2i), lowers BMD and increases fracture risk compared with the respective outcomes in the placebo group [10]. However, other SGLT2i, dapagliflozin or empagliflozin, did not report a decrease in bone density or an increase in fractures [11]. Alternatively, metformin and sulfonylurea have a neutral effect on bone, while incretin-based treatments may have a positive effect [5]. Most studies to date have performed comparisons with placebo or between single drugs; however, in practice, it is more common to combine multiple antidiabetic agents with different mechanisms to lower blood glucose than to administer a single agent. Moreover, data on the effects of gemigliptin and empagliflozin on bone metabolism in real practice are limited. Therefore, in this prospective study, patients who had previously been treated with various regimens of oral combination therapy were divided into groups, changes in bone mass and bone markers were prospectively observed for 12 months, and differences between groups were compared.

\section{METHODS}

\section{Patients and sample collection}

We conducted a prospective, multicenter, open-label, comparative study to determine the effects of antidiabetic medications on bone metabolism in patients with T2DM. The study flow for study subject evaluation is depicted in Fig. 1. Subjects with T2DM who had previously been treated with metformin monotherapy or combination metformin/sulfonylurea were divided into four groups ( $n=227)$ : Group 1, continued metformin monotherapy or combination metformin/sulfonylurea $(n=52)$; Group 2 , addition of thiazolidinedione (TZD) $(n=60)$; Group 3, addition of dipeptidyl peptidase-4 inhibitor (DPP4i) $(n=61)$; and Group 4, addition of SGLT2i $(n=54)$. BMD and bone turnover markers (BTMs) were prospectively observed for 12 months (Fig. 1). After exclusion, a total of 193 subjects ( 45 subjects in Group 1, 47 subjects in Group 2, 53 subjects in Group 3, and 48 subjects in Group 4) were eligible for the analysis (Fig. 1). The type and dosage of medications were determined according to the physician's clinical discretion. Subjects with other underlying diseases that would affect bone metabolism were excluded from the enrollment. Subjects were excluded if they were treated with any of the following drugs: corticosteroid, anticonvulsants, thyroid hormones, aromatase inhibitors, bisphosphonates, estrogen or selective estrogen receptor modulator, parathyroid hormone (PTH), teriparatide, or denosumab. For sulfonylureas, glimepiride was employed, with daily doses between 0.5 and $2 \mathrm{mg}$. Daily metformin titration ranged from 500 to $1,500 \mathrm{mg}$, depending on the physician's discretion. Among TZDs, pioglitazone account- 


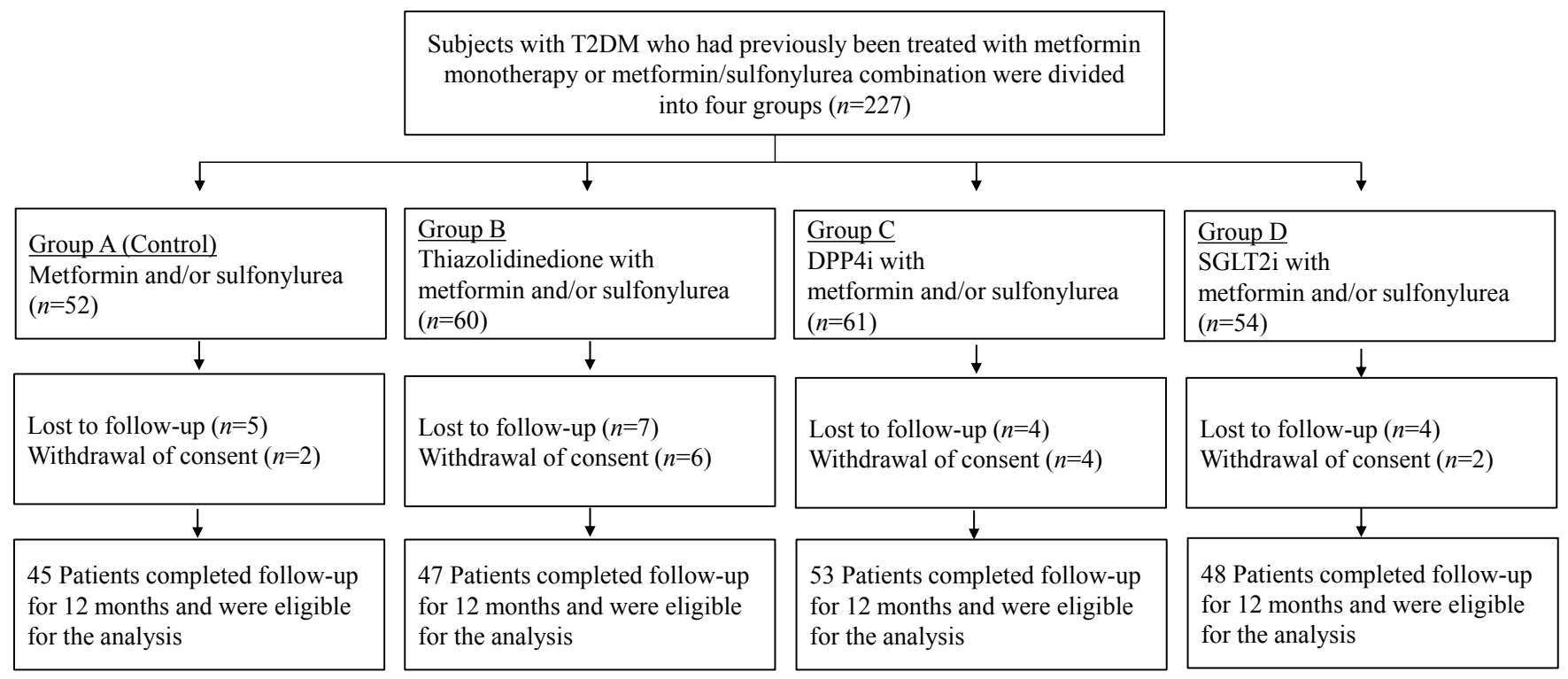

Fig. 1. Enrollment, randomization, and follow-up of participants. T2DM, type 2 diabetes mellitus; DPP4i, dipeptidyl peptidase-4 inhibitor; SGLT2i, sodium-glucose cotransporter 2 inhibitor.

ed for $76.6 \%$ and lobeglitazone accounted for $23.4 \%$. Gemigliptin $50 \mathrm{mg}$ was administered as a DPP4i, and empagliflozin 10 or $25 \mathrm{mg}$ was administered as an SGLT2i. All study subjects received calcium and vitamin $\mathrm{D}$ supplements, with the aim of maintaining serum 25-hydroxyvitamin D levels $\geq 20 \mathrm{ng} / \mathrm{mL}$. Performing moderate-intensity exercise for 150 minutes per week was recommended to all study participants. For the analysis, patients in each group were prospectively observed for 12 months, and changes in BMD and biochemical markers, including BTMs, were evaluated. BMD was measured at baseline and 12 months. Subjects' body weight and height were simultaneously measured while measuring BMD. Blood was sampled between 7:00 AM and 9:00 AM after 8 hours of overnight fasting to evaluate serum glucose, calcium, phosphorus, creatinine, albumin, PTH, 25-hydroxyvitamin $\mathrm{D}$, total procollagen type 1 amino-terminal propeptide (P1NP), and C-terminal type 1 collagen telopeptide (CTx). Biochemical testing, except BTM measurement, was performed five times during the study period: at baseline, 3, 6, 9, and 12 months. BTM was measured three times: at baseline, between 3 and 6 months of the study, and between 9 and 12 months of the study. Serum calcium was corrected for changes in serum albumin concentration according to the following formula: corrected calcium $(\mathrm{mg} / \mathrm{dL})=$ actual calcium $+(4-$ serum albumin $) \times 0.8$. As a part of standard care monitoring, glycated hemoglobin (HbA1c) and fasting serum glucose were measured at every follow-up to provide a standard of care for patients with diabetes. This study protocol was re- viewed and approved by the Institutional Review Board of Yeouido St. Mary's Hospital (Seoul, Korea), and all patients signed an informed consent form before entering the study (XC16OIMI0031S).

\section{Assays}

Serum calcium, phosphorus, albumin, and creatinine levels were determined using an autoanalyzer (747 automatic analyzer, Hitachi, Tokyo, Japan). HbA1c levels were measured using high-performance liquid chromatography (HLC- 723G7, Tosoh, Tokyo, Japan). Serum P1NP (Elecsys total P1NP, Roche Diagnostics, Rotkreuz, Switzerland), CTx (Elecsys B-CrossLaps, Roche Diagnostics), and PTH (intact PTH, Elecsys PTH, Roche Diagnostics) concentrations were determined in duplicate using an ECLIA (Cobas e 801, Roche Diagnostics). 25-Hydroxyvitamin D (Access 25(OH) Vitamin D Total DXI reagent, Beckman Coulter Inc., Brea, CA, USA) was measured using a UniCel DxI 800 Immunoassay Analyzer (Beckman Coulter Inc.). The maximum inter- and intra-assay coefficients of variation $(\mathrm{CVs})$ for the range of concentrations evaluated were $4.1 \%$ and $2.6 \%$ for P1NP and $6.5 \%$ and $3.6 \%$ for CTx. The manufacturer suggested a reference range for $\mathrm{CTx}$ in postmenopausal women $<1.008 \mathrm{ng} / \mathrm{mL}$ and a reference range for P1NP in postmenopausal women without postmenopausal hormone replacement therapy of 20.25 to $76.31 \mathrm{ng} / \mathrm{mL}$. 


\section{BMD measurement}

The BMD of the lumbar spine (lumbar vertebrae L1-L4), femoral neck, and total hip were measured by dual-energy X-ray absorptiometry using a Hologic Delphi W (Hologic Inc., Bedford, MA, USA) at baseline and after 12 months. The CV was determined to be $1.2 \%$ at the lumbar spine and $1.9 \%$ at the femoral neck. The BMD measurement was performed by one technician with $>10$ years of experience. To properly control the quality of bone density measurement, our institution performed quality control for the bone density equipment and the bone density tester according to the protocol.

\section{Statistical analysis}

All data were analyzed using SPSS version 20.0 for Windows (IBM Co., Armonk, NY, USA). The data are presented as the mean \pm standard deviation, unless otherwise stated. Graphics were produced using GraphPad Prism version 5.0 (GraphPad
Software Inc., San Diego, CA, USA). In the evaluation of clinical features, we used the chi-square test for analysis of categorical variables and Student's $t$ test for the analysis of continuous variables. The mean percentage changes for BMD and other biochemical markers from baseline were analyzed using repeated measures analysis of variation (ANOVA) and Dunnett's method, if appropriate. $P$ values $<0.05$ were considered to represent statistically significant results for all comparisons.

\section{RESULTS}

\section{Baseline characteristics}

Clinical characteristics at baseline are summarized in Table 1. The subjects were postmenopausal women with a mean age of $66.5 \pm 7.1$ years and experienced menopause an average of $16.1 \pm$ 8.0 years prior. There was no difference between the four groups in regard to age, years after menopause, or body mass index. The

Table 1. Baseline Characteristics of the Study Population by Group

\begin{tabular}{|c|c|c|c|c|c|c|}
\hline Characteristic & Total $(n=193)$ & Group $1(n=45)$ & Group $2(n=47)$ & Group $3(n=53)$ & Group $4(n=48)$ & $P$ value \\
\hline Age, yr & $66.5 \pm 7.1$ & $65.4 \pm 7.0$ & $65.9 \pm 7.0$ & $67.9 \pm 7.2$ & $66.5 \pm 7.0$ & 0.309 \\
\hline Years since menopause & $16.1 \pm 8.0$ & $17.1 \pm 9.4$ & $15.5 \pm 7.2$ & $17.4 \pm 8.6$ & $14.1 \pm 6.4$ & 0.277 \\
\hline Height, cm & $153.2 \pm 12.0$ & $153.4 \pm 4.9$ & $153.7 \pm 4.8$ & $151.6 \pm 3.6$ & $154.5 \pm 4.4$ & 0.640 \\
\hline Weight, kg & $60.0 \pm 7.8$ & $59.8 \pm 7.2$ & $60.5 \pm 8.2$ & $59.4 \pm 8.2$ & $60.5 \pm 7.4$ & 0.473 \\
\hline Body mass index, $\mathrm{kg} / \mathrm{m}^{2}$ & $25.3 \pm 3.0$ & $25.7 \pm 3.0$ & $25.6 \pm 3.1$ & $24.9 \pm 3.1$ & $25.4 \pm 2.8$ & 0.321 \\
\hline Diabetes duration, yr & $10.4 \pm 7.9$ & $8.0 \pm 6.5$ & $11.5 \pm 7.7$ & $11.5 \pm 7.7$ & $10.5 \pm 9.2$ & 0.820 \\
\hline Glycated hemoglobin, \% & $6.5 \pm 0.6$ & $6.5 \pm 0.5$ & $6.5 \pm 0.6$ & $6.4 \pm 0.5$ & $6.6 \pm 0.6$ & 0.184 \\
\hline Previous clinical fracture & $16(8.3)$ & $4(8.9)$ & $3(6.4)$ & $5(9.4)$ & $4(8.3)$ & 0.439 \\
\hline \multicolumn{7}{|l|}{ T-score } \\
\hline Lumbar spine & $-1.6 \pm 1.1$ & $-1.8 \pm 1.1$ & $-1.7 \pm 1.2$ & $-1.7 \pm 1.0$ & $-1.3 \pm 1.1$ & 0.199 \\
\hline Femoral neck & $-1.3 \pm 0.9$ & $-1.3 \pm 1.0$ & $-1.3 \pm 0.8$ & $-1.3 \pm 0.9$ & $-1.2 \pm 0.8$ & 0.959 \\
\hline Total hip & $-0.7 \pm 1.0$ & $-0.8 \pm 1.1$ & $-0.8 \pm 1.0$ & $-0.8 \pm 1.0$ & $-0.6 \pm 0.9$ & 0.719 \\
\hline \multicolumn{7}{|l|}{$\mathrm{BMD}, \mathrm{g} / \mathrm{cm}^{2}$} \\
\hline Lumbar spine & $0.942 \pm 0.132$ & $0.928 \pm 0.129$ & $0.932 \pm 0.142$ & $0.932 \pm 0.127$ & $0.977 \pm 0.131$ & 0.226 \\
\hline Femoral neck & $0.790 \pm 0.104$ & $0.790 \pm 0.121$ & $0.790 \pm 0.095$ & $0.784 \pm 0.103$ & $0.795 \pm 0.100$ & 0.953 \\
\hline Total hip & $0.887 \pm 0.120$ & $0.881 \pm 0.126$ & $0.883 \pm 0.120$ & $0.883 \pm 0.120$ & $0.905 \pm 0.112$ & 0.681 \\
\hline Serum CTx, ng/mL & $0.44 \pm 0.20$ & $0.44 \pm 0.22$ & $0.42 \pm 0.17$ & $0.43 \pm 0.20$ & $0.49 \pm 0.22$ & 0.385 \\
\hline Serum P1NP, ng/mL & $47.2 \pm 16.6$ & $49.1 \pm 16.7$ & $45.3 \pm 11.8$ & $45.2 \pm 15.3$ & $49.5 \pm 21.9$ & 0.509 \\
\hline Serum 25-hydroxyvitamin D, ng/mL & $25.9 \pm 7.5$ & $25.8 \pm 6.6$ & $25.4 \pm 9.3$ & $26.0 \pm 7.2$ & $26.4 \pm 6.8$ & 0.928 \\
\hline Serum calcium, mg/dL & $9.2 \pm 0.4$ & $9.2 \pm 0.4$ & $9.1 \pm 0.3$ & $9.2 \pm 0.4$ & $9.2 \pm 0.4$ & 0.510 \\
\hline Serum creatinine, $\mathrm{mg} / \mathrm{dL}$ & $0.67 \pm 0.16$ & $0.63 \pm 0.14$ & $0.74 \pm 0.23$ & $0.67 \pm 0.13$ & $0.65 \pm 0.14$ & 0.097 \\
\hline
\end{tabular}

Values are expressed as mean \pm standard deviation or number (\%). Group 1, metformin or metformin/sulfonylurea combination; Group 2, thiazolidinedione with metformin and/or sulfonylurea combination; Group 3, dipeptidyl peptidase-4 inhibitor (gemigliptin) with metformin and/or sulfonylurea combination; Group 4, sodium-glucose cotransporter 2 inhibitor (empagliflozin) with metformin and/or sulfonylurea combination.

$\mathrm{BMD}$, bone mineral density; CTx, C-terminal telopeptides of type I collagen; P1NP, total procollagen type 1 amino-terminal propeptide. 
mean duration of diabetes was $10.4 \pm 7.9$ years, with Group 1 having the shortest duration at $8.0 \pm 6.5$ years, but there was no significant difference among the groups. The mean baseline $\mathrm{HbA1c}$ level was $6.5 \% \pm 0.6 \%$, without significant differences among groups. The baseline BMD was $0.942 \pm 0.132$ for the lumbar spine, $0.790 \pm 0.104$ for the femoral neck, and $0.887 \pm 0.120$ for the total hip, and T-score at baseline was $-1.6 \pm 1.1$. There was no significant difference in the T-score or BMD at baseline for all measurement sites among the groups. In addition, detailed information on the prescription patterns of each group is provided in Table 2. In each group, a dual combination with metformin was the most commonly prescribed combination.

Table 2. Prescription Patterns of Antidiabetic Medications in Each Group

\begin{tabular}{llc}
\hline & \multicolumn{1}{c}{ Patterns } & No. (\%) \\
\hline Group 1 $(n=45)$ & Metformin+Sulfonylurea & $41(91.1)$ \\
& Metformin monotherapy & $4(8.9)$ \\
Group 2 $(n=47))$ & Metformin+Thiazolidinedione & $38(80.9)$ \\
& Metformin+Thiazolidinedione+ & $9(19.1)$ \\
& Sulfonylurea & \\
Group 3 $(n=53)$ & Metformin+DPP4i & $43(81.1)$ \\
& Metformin+DPP4i+Sulfonylurea & $10(18.9)$ \\
Group 4 $(n=48)$ & Metformin+SGLT2i & $38(79.2)$ \\
& Metformin+SGLT2i+Sulfonylurea & $10(20.8)$ \\
\hline
\end{tabular}

Group 1, metformin or metformin/sulfonylurea combination; Group 2, thiazolidinedione with metformin and/or sulfonylurea combination, pioglitazone accounted for $76.6 \%$ and lobeglitazone accounted for $23.4 \%$; Group 3, DPP4i (gemigliptin) with metformin and/or sulfonylurea combination; Group 4, SGLT2i (empagliflozin) with metformin and/or sulfonylurea combination.

DPP4i, dipeptidyl peptidase-4 inhibitor; SGLT2i, sodium-glucose cotransporter 2 inhibitor.

\section{Changes in BMD}

The percentage changes in BMD from baseline to 12 months were analyzed according to groups (Table 3, Fig. 2). The lumbar spine BMD reduction was found to be the highest in the SGLT2i group (Group 4) $(-1.28 \% \pm 2.65 \%)$, despite not exhibiting a significant decrease compared to baseline $(P=0.314)$ or a statistically significant difference among groups $(P=0.265)$. The decrease in femoral neck BMD in the TZD group (Group 2) at 12 months was found to be significant compared to the baseline value $(-2.50 \% \pm 3.08 \%, P=0.015)$, and the reduction was also significant in comparison with the other three groups $(P=0.012)$. For the total hip, BMD decreased significantly from baseline at 12 months in the TZD group (Group 2) $(-1.74 \% \pm 1.48 \%, P=$ 0.046 ) and was also significantly different from sulfonylurea

Table 3. Difference in Percentage Changes of Bone Mineral Density from Baseline to 12 Months between Groups

\begin{tabular}{|c|c|c|c|}
\hline & \multicolumn{2}{|c|}{ Lumbar spine, $\%$ Femoral neck, $\%$} & Total hip, $\%$ \\
\hline Group 1 & $-0.24 \pm 2.32$ & $-0.79 \pm 2.86$ & $-0.57 \pm 1.79$ \\
\hline Group 2 & $-1.00 \pm 2.94$ & $-2.50 \pm 3.08^{\mathrm{a}}$ & $-1.74 \pm 1.48^{\mathrm{b}}$ \\
\hline Group 3 & $-0.62 \pm 2.50$ & $-1.05 \pm 2.74$ & $-0.75 \pm 1.87$ \\
\hline Group 4 & $-1.28 \pm 2.65$ & $-1.24 \pm 2.91$ & $-1.27 \pm 1.72$ \\
\hline
\end{tabular}

Values are expressed as mean \pm standard deviation. Group 1, metformin or metformin/sulfonylurea combination; Group 2, thiazolidinedione with metformin and/or sulfonylurea combination; Group 3, dipeptidyl peptidase-4 inhibitor (gemigliptin) with metformin and/or sulfonylurea combination; Group 4, sodium-glucose cotransporter 2 inhibitor (empagliflozin) with metformin and/or sulfonylurea combination. $P$ for trend compared with the baseline; $P$ value by one-way analysis of variation (ANOVA) and post hoc analysis. Dunnett's method was applied for post hoc analysis.

${ }^{\mathrm{a}} P<0.05$ compared to baseline and other groups; ${ }^{\mathrm{b}} P<0.05$ compared to baseline and Group 1 and 3 .

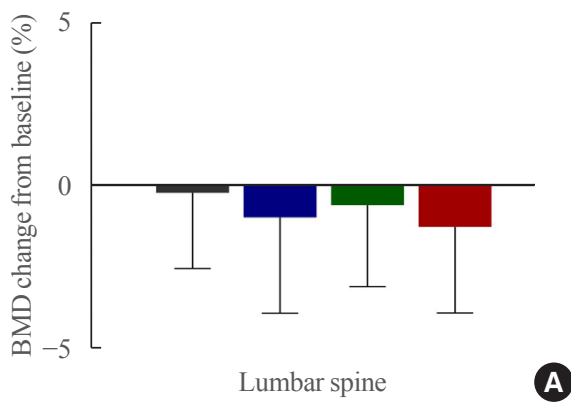

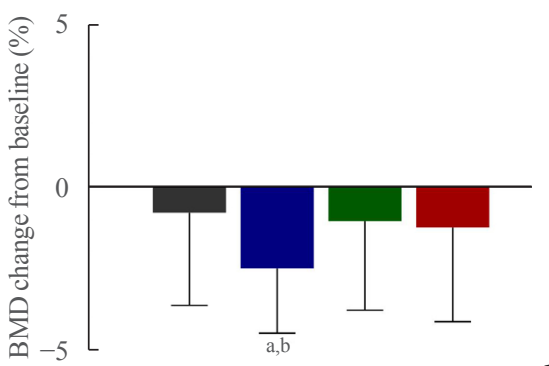

Femoral neck

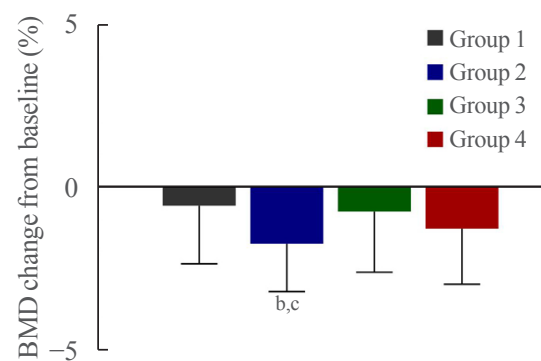

Total hip

Fig. 2. Percentage change of bone mineral density (BMD) from baseline to 12 months. (A) Lumbar spine, (B) femoral neck, (C) total hip. Group 1, metformin or metformin/sulfonylurea combination; Group 2, thiazolidinedione with metformin and/or sulfonylurea combination; Group 3, dipeptidyl peptidase 4 inhibitor with metformin and/or sulfonylurea combination; Group 4, sodium-glucose cotransporter 2 inhibitors with metformin and/or sulfonylurea combination. ${ }^{\text {a }} P<0.05$ compared with Group 1, 3, and 4; ${ }^{b} P<0.05$ compared with baseline; ${ }^{\mathrm{c}} P<0.05$ compared with Group 1 and 3 . 
Table 4. Bone Turnover Markers during the Study Period

\begin{tabular}{ccccc}
\hline & Baseline & Point 1 & Point 2 & $P$ for trend \\
\hline CTx, ng/mL & & & & \\
Group 1 & $0.44 \pm 0.22$ & $0.50 \pm 0.25$ & $0.45 \pm 0.22$ & 0.368 \\
Group 2 & $0.42 \pm 0.17$ & $0.43 \pm 0.16$ & $0.43 \pm 0.16$ & 0.713 \\
Group 3 & $0.43 \pm 0.20$ & $0.45 \pm 0.17$ & $0.44 \pm 0.17$ & 0.215 \\
Group 4 & $0.49 \pm 0.22$ & $0.48 \pm 0.17$ & $0.47 \pm 0.20$ & 0.709 \\
P1NP, ng/mL & & & & \\
Group 1 & $49.1 \pm 16.7$ & $52.1 \pm 13.8$ & $51.5 \pm 18.6$ & 0.448 \\
Group 2 & $45.3 \pm 11.8$ & $47.2 \pm 13.9$ & $47.1 \pm 12.4$ & 0.543 \\
Group 3 & $45.2 \pm 15.3$ & $47.9 \pm 15.5$ & $45.7 \pm 15.4$ & 0.198 \\
Group 4 & $49.5 \pm 21.9$ & $51.6 \pm 18.2$ & $50.4 \pm 18.7$ & 0.253 \\
\hline
\end{tabular}

Values are expressed as mean \pm standard deviation. Point 1, between 3-6 months of the study; Point 2, between 9-12 months of the study. Group 1, metformin or metformin/sulfonylurea combination; Group 2, thiazolidinedione with metformin and/or sulfonylurea combination; Group 3 , dipeptidyl peptidase-4 inhibitor (gemigliptin) with metformin and/or sulfonylurea combination; Group 4, sodium-glucose cotransporter 2 inhibitor (empagliflozin) with metformin and/or sulfonylurea combination. $P$ for trend compared with the baseline; $P$ value by one-way analysis of variation (ANOVA) and post hoc analysis. Dunnett's method was applied for post hoc analysis.

CTx, C-terminal type 1 collagen telopeptide; P1NP, total procollagen type 1 amino-terminal propeptide.

group (Group 1) $(P=0.007)$ and the DPP4i group (Group 3) $(P=0.024)$ but not from the SGLT2i group (Group 4) $(P=0.638)$.

\section{Levels of bone turnover markers}

Serum levels of CTx and P1NP during the study period are summarized in Table 4. No significant changes in CTx or P1NP were observed during the study period in any group. During the 9- to 12-month period of the study, the lowest CTx was measured in the TZD group (Group 2) $(0.43 \pm 0.16 \mathrm{ng} / \mathrm{mL})$, but it was not significantly different from the other groups. In all groups, CTx was maintained below $1.008 \mathrm{ng} / \mathrm{mL}$, the reference range for postmenopausal women, and P1NP was also maintained between 20.25 and $76.31 \mathrm{ng} / \mathrm{mL}$, the reference range for postmenopausal women without postmenopausal hormone replacement therapy.

\section{Metabolic parameters}

During the study period, weight gain in the TZD group and weight loss in the SGLT2i group were seen; however, the change was not significant, and the final weight was not statistically different between the four groups. No significant changes in $\mathrm{HbA1c}$ levels were observed during the study period. At 12 months, $\mathrm{HbA} 1 \mathrm{c}$ was $6.4 \% \pm 0.5 \%$ for Group $1,6.4 \% \pm 0.8 \%$ for Group 2, $6.4 \% \pm 0.6 \%$ for Group 3, and $6.7 \% \pm 0.8 \%$ for Group 4. Neither differences between groups nor differences within groups were significant.

\section{Adverse events and safety}

In the TZD group (Group 2), three (6.4\%) patients reported peripheral edema, but none stopped taking medication for this reason. In the SGLT2i group (Group 4), urinary tract infection was reported in two patients $(4.2 \%)$, and genital infection was reported in one patient $(2.1 \%)$. No symptomatic hypoglycemia was reported in any of the four groups during the study period. No serious adverse events resulting in discontinuation of the medication were reported in any group during the study period.

\section{DISCUSSION}

In this prospective study, significant reductions in femoral neck and total hip BMD were confirmed in postmenopausal T2DM patients treated with combination therapy with TZD. The femoral neck was the site with the largest decrease $(-2.50 \% \pm 3.08 \%)$ compared to the baseline level, which was also significantly higher than the levels in the other groups. Administration of combination regimens containing empagliflozin, an SGLT2i, did not cause significant bone loss.

TZDs are an antidiabetic medication that improves insulin sensitivity, but their use is associated with reduced bone mass $[12,13]$ and increased risk for fracture [14]. However, lobeglitazone showed no detrimental effect on osteoblast differentiation in vitro [15]. An important mechanistic explanation for the effect of TZDs on bone is that TZDs induce adipogenesis and inhibits regulators of bone differentiation [16]. A meta-analysis of 22 randomized controlled trials concluded that pioglitazone treatment is associated with an increased risk of fractures in women (odds ratio of 1.73) [17]. Schwartz et al. [13] reported that TZD treatment, namely, pioglitazone, may cause bone loss in the whole body of older women with T2DM. Similar results were found in a study of nondiabetic subjects. In a large randomized controlled trial, pioglitazone was associated with a significant reduction in the risk of stroke and myocardial infarction in nondiabetic patients with cerebrovascular disease but was associated with an increased fracture risk [9]. Most previous studies have compared pioglitazone with placebo or with a single comparative agent. However, in an actual clinical environment, not only pioglitazone monotherapy but also combination therapy containing pioglitazone are prescribed for T2DM treatment. 
In this study, the harmful effect of TZD monotherapy on bone metabolism was also reproduced in the group treated with combination therapy containing TZD. Despite the great advantages of TZD for the treatment of T2DM, the benefits and harm of pioglitazone treatment must be carefully considered in T2DM patients at a high risk of fracture.

SGLT2i block glucose reabsorption in the proximal kidney tubule by blocking sodium-glucose cotransporters, resulting in a lower serum glucose concentration [18]. Dapagliflozin, empaglifozin, and canagliflozin are agents belonging to this class and have common characteristics of lowering blood glucose and preventing cardiovascular disease but seem to have different effects on bone metabolism. In a recent randomized controlled trial, canagliflozin was shown to be associated with a decrease in total hip BMD ( $-1.2 \%$ of placebo-subtracted changes in canagliflozin $300 \mathrm{mg}$ ) and an increase in CTx and osteocalcin over 104 weeks [19]. Blau et al. [20] suggested that canagliflozin has adverse effects on bone because it induces an immediate increase in serum phosphorous, causing downstream changes in fibroblast growth factor-23, 1,25-dihydroxyvitamin D, and PTH. However, studies on dapagliflozin and empagliflozin (mostly dapagliflozin) have reported neutral effects on BMD, BTM, and risk of fracture [5,21,22]. Although these agents belong to the same SGLT2 $\mathrm{i}$ class, the physiological mechanism leading to different bone safety profiles has not yet been elucidated. We found that total hip BMD decreased by $1.27 \% \pm$ $1.72 \%$ during the 12-month study period in Group 4 treated with empagliflozin, but this was not a significant decrease compared to the baseline value. There have been few studies on the effects of empagliflozin on bone metabolism, and our studies have shown that empagliflozin does not cause significant bone loss, as observed in the TZD group. Moreover, since the combination of empagliflozin and metformin accounted for most of the prescriptions in Group 4, it realistically reflects the current T2DM treatment in which metformin is administered as a firstline agent. Currently, canagliflozin is not available in Korea. A prospective comparative study of SGLT2i, including canagliflozin, will help to elucidate the effect of SGLT2i on bone metabolism more clearly.

In T2DM patients, BTM levels are altered. Based on the pooled data in a meta-analysis including 22 studies, CTx and osteocalcin were significantly lower in patients with diabetes than in the nondiabetic population [23]. Farr et al. [24] reported significantly lower serum CTx and serum P1NP levels in 30 postmenopausal patients with T2DM durations $>10$ years. Consistent with previous reports [24-27], our analysis revealed that
BTM levels were lowered in T2DM patients. However, in our study, there was no nondiabetic control, but BTM levels were maintained at lower than the reference value in postmenopausal women. The mechanisms for reduced BTM, in particular, reduced bone formation in T2DM patients, are not well understood. T2DM may deteriorate bone homeostasis by suppressing osteoblast differentiation and accelerating osteoblast apoptosis, which is expressed as a reduced formation marker [28]. Advanced glycation end-products (AGEs) and oxidative stress are identified as possible causes of BTM changes. In T2DM patients with prolonged exposure to high glucose concentrations, more AGE accumulates in bone tissue, which negatively affects bone quality, resulting in fragile bones [28-30]. BTM changes after administration of a single agent have been differently reported. With TZD treatment, bone resorption markers increase but formation markers decrease, while both resorption and formation markers increase with SGLT2i [31]. Furthermore, DPP4i and sulfonylurea have a neutral effect on changes in bone resorption markers [31], whereas metformin decreased serum CTx/P1NP levels in the ADOPT trial [32]. The effect of a combination therapy on BTM is expected to show varied patterns, but clear evidence is lacking. In our study, we intended to determine the change in BTM levels under various combination regimens, and we found that BTM levels were not altered during the 12-month study period in any of the four groups. However, it is difficult to provide a clear basis for these results.

Our study has several limitations. First, the duration of diabetes was shorter in Group 1 than in the other groups. Guidelines recommend metformin as the first-line therapy in the treatment of T2DM patients unless it is contraindicated [33,34], and Group 1 includes patients undergoing metformin monotherapy, resulting in a relatively short duration of disease. However, no significant difference was observed between groups in regard to the duration of diabetes. Second, there was no quantitative analysis of dietary calcium intake. However, elemental calcium (250 $\mathrm{mg}$ of calcium carbonate) and cholecalciferol (1,000 IU) were administered to all subjects, and serum 25-hydroxyvitamin D levels were maintained at $\geq 20 \mathrm{ng} / \mathrm{mL}$ during the study period, with no difference among the four groups. Another drawback is that the prescription of diabetic medication was heterogeneous. Our study included participants with various prescriptions from monotherapy to dual and triple combination, and it may be difficult to conclude that the result represents the specific effect of a distinct medication. With metformin as the baseline treatment, the dual combinations of sulfonylurea (Group 1), TZD (mainly pioglitazone) (Group 2), gemigliptin (Group 3), and empa- 
gliflozin (Group 4) were the most prescribed patterns in each group. Therefore, the results primarily represent the effect of the dual combination with metformin. Lastly, it was difficult to identify new fractures during the study period due to the short study period and the limited number of study subjects.

This is the first study to compare the effects of combination therapy on BMD and BTM based on previous findings on the bone-related effects of antidiabetic agents. To regulate the various pathophysiological mechanisms of diabetes, it is recommended to administer a combination of antidiabetic agents from the early stages of the disease [35]. As prevalence of combination therapy rather than a single drug prescription continues to increase, careful attention is needed on how combination therapy affects BMD and BTM. In conclusion, patients on combination therapy with TZD exhibited noticeable cortical bone loss. On the other hand, in patients treated with empagliflozin (SGLT2i) combination, gemigliptin (DPP4i) combination, or sulfonylurea combination, bone loss was not significant at any measurement site during the 12-month follow-up period. Thus, caution should be exercised during treatment with antidiabetic medications or combinations that adversely affect bone in patients with a high risk of bone loss.

\section{CONFLICTS OF INTEREST}

This research was funded by LG Chem, LTD. (Seoul, Korea). The funders had no role in the study design, data collection and analysis, decision to publish, or preparation of the manuscript.

\section{ACKNOWLEDGMENTS}

This work was supported by the Korean Endocrine Society of EnM Research Award 2020.

\section{AUTHOR CONTRIBUTIONS}

Conception or design: H.S.K., S.H.K., S.D.M., K.H.B. Acquisition, analysis, or interpretation of data: J.H., Y.L., M.K.K. Drafting the work or revising: J.H., K.H.B. Final approval of the manuscript: K.H.S., M.I.K., K.H.B.

\section{ORCID}

Jeonghoon Ha https://orcid.org/0000-0001-9219-7135

Sung Dae Moon https://orcid.org/0000-0002-3094-9415

Ki-Hyun Baek https://orcid.org/0000-0002-4097-1077

\section{REFERENCES}

1. Assessment of fracture risk and its application to screening for postmenopausal osteoporosis. Report of a WHO Study Group. World Health Organ Tech Rep Ser 1994;843:1-129.

2. Burge R, Dawson-Hughes B, Solomon DH, Wong JB, King A, Tosteson A. Incidence and economic burden of osteoporosis-related fractures in the United States, 2005-2025. J Bone Miner Res 2007;22:465-75.

3. Aziziyeh R, Amin M, Habib M, Garcia Perlaza J, Szafranski $\mathrm{K}$, McTavish RK, et al. The burden of osteoporosis in four Latin American countries: Brazil, Mexico, Colombia, and Argentina. J Med Econ 2019;22:638-44.

4. Mohd-Tahir NA, Li SC. Economic burden of osteoporosisrelated hip fracture in Asia: a systematic review. Osteoporos Int 2017;28:2035-44.

5. Napoli N, Chandran M, Pierroz DD, Abrahamsen B, Schwartz AV, Ferrari SL, et al. Mechanisms of diabetes mellitus-induced bone fragility. Nat Rev Endocrinol 2017;13:20819.

6. Vestergaard P. Discrepancies in bone mineral density and fracture risk in patients with type 1 and type 2 diabetes: a meta-analysis. Osteoporos Int 2007;18:427-44.

7. Schwartz AV, Vittinghoff E, Bauer DC, Hillier TA, Strotmeyer ES, Ensrud KE, et al. Association of BMD and FRAX score with risk of fracture in older adults with type 2 diabetes. JAMA 2011;305:2184-92.

8. Bonds DE, Larson JC, Schwartz AV, Strotmeyer ES, Robbins J, Rodriguez BL, et al. Risk of fracture in women with type 2 diabetes: the Women's Health Initiative Observational Study. J Clin Endocrinol Metab 2006;91:3404-10.

9. Viscoli CM, Inzucchi SE, Young LH, Insogna KL, Conwit R, Furie KL, et al. Pioglitazone and risk for bone fracture: safety data from a randomized clinical trial. J Clin Endocrinol Metab 2017;102:914-22.

10. Watts NB, Bilezikian JP, Usiskin K, Edwards R, Desai M, Law $\mathrm{G}$, et al. Effects of canagliflozin on fracture risk in patients with type 2 diabetes mellitus. J Clin Endocrinol Metab 2016;101:157-66.

11. Fitchett D. A safety update on sodium glucose co-transporter 2 inhibitors. Diabetes Obes Metab 2019;21 Suppl 2:34-42.

12. Grey A, Bolland M, Gamble G, Wattie D, Horne A, Davidson $\mathrm{J}$, et al. The peroxisome proliferator-activated receptorgamma agonist rosiglitazone decreases bone formation and bone mineral density in healthy postmenopausal women: a randomized, controlled trial. J Clin Endocrinol Metab 2007; 
92:1305-10.

13. Schwartz AV, Sellmeyer DE, Vittinghoff E, Palermo L, Lecka-Czernik B, Feingold KR, et al. Thiazolidinedione use and bone loss in older diabetic adults. J Clin Endocrinol Metab 2006;91:3349-54.

14. Kahn SE, Haffner SM, Heise MA, Herman WH, Holman RR, Jones NP, et al. Glycemic durability of rosiglitazone, metformin, or glyburide monotherapy. N Engl J Med 2006; 355:2427-43.

15. Kim KM, Jin HJ, Lee SY, Maeng HJ, Lee GY, Oh TJ, et al. Effects of lobeglitazone, a new thiazolidinedione, on osteoblastogenesis and bone mineral density in mice. Endocrinol Metab (Seoul) 2017;32:389-95.

16. Benvenuti S, Cellai I, Luciani P, Deledda C, Baglioni S, Giuliani $\mathrm{C}$, et al. Rosiglitazone stimulates adipogenesis and decreases osteoblastogenesis in human mesenchymal stem cells. J Endocrinol Invest 2007;30:RC26-30.

17. Zhu ZN, Jiang YF, Ding T. Risk of fracture with thiazolidinediones: an updated meta-analysis of randomized clinical trials. Bone 2014;68:115-23.

18. Tahrani AA, Barnett AH, Bailey CJ. Pharmacology and therapeutic implications of current drugs for type 2 diabetes mellitus. Nat Rev Endocrinol 2016;12:566-92.

19. Bilezikian JP, Watts NB, Usiskin K, Polidori D, Fung A, Sullivan D, et al. Evaluation of bone mineral density and bone biomarkers in patients with type 2 diabetes treated with canagliflozin. J Clin Endocrinol Metab 2016;101:44-51.

20. Blau JE, Bauman V, Conway EM, Piaggi P, Walter MF, Wright EC, et al. Canagliflozin triggers the FGF23/1,25-dihydroxyvitamin D/PTH axis in healthy volunteers in a randomized crossover study. JCI Insight 2018;3:e99123.

21. Tang HL, Li DD, Zhang JJ, Hsu YH, Wang TS, Zhai SD, et al. Lack of evidence for a harmful effect of sodium-glucose co-transporter 2 (SGLT2) inhibitors on fracture risk among type 2 diabetes patients: a network and cumulative metaanalysis of randomized controlled trials. Diabetes Obes Metab 2016;18:1199-206.

22. Ljunggren O, Bolinder J, Johansson L, Wilding J, Langkilde AM, Sjostrom CD, et al. Dapagliflozin has no effect on markers of bone formation and resorption or bone mineral density in patients with inadequately controlled type 2 diabetes mellitus on metformin. Diabetes Obes Metab 2012;14: 990-9.

23. Starup-Linde J, Eriksen SA, Lykkeboe S, Handberg A, Vestergaard P. Biochemical markers of bone turnover in diabetes patients: a meta-analysis, and a methodological study on the effects of glucose on bone markers. Osteoporos Int
2014;25:1697-708.

24. Farr JN, Drake MT, Amin S, Melton LJ 3rd, McCready LK, Khosla S. In vivo assessment of bone quality in postmenopausal women with type 2 diabetes. J Bone Miner Res 2014; 29:787-95.

25. Gerdhem P, Isaksson A, Akesson K, Obrant KJ. Increased bone density and decreased bone turnover, but no evident alteration of fracture susceptibility in elderly women with diabetes mellitus. Osteoporos Int 2005;16:1506-12.

26. Krakauer JC, McKenna MJ, Buderer NF, Rao DS, Whitehouse FW, Parfitt AM. Bone loss and bone turnover in diabetes. Diabetes 1995;44:775-82.

27. Manavalan JS, Cremers S, Dempster DW, Zhou H, Dworakowski E, Kode A, et al. Circulating osteogenic precursor cells in type 2 diabetes mellitus. J Clin Endocrinol Metab 2012;97:3240-50.

28. Manolagas SC. From estrogen-centric to aging and oxidative stress: a revised perspective of the pathogenesis of osteoporosis. Endocr Rev 2010;31:266-300.

29. Leslie WD, Rubin MR, Schwartz AV, Kanis JA. Type 2 diabetes and bone. J Bone Miner Res 2012;27:2231-7.

30. Starup-Linde J, Hygum K, Langdahl BL. Skeletal fragility in type 2 diabetes mellitus. Endocrinol Metab (Seoul) 2018; 33:339-51.

31. Vianna AGD, Sanches CP, Barreto FC. Review article: effects of type 2 diabetes therapies on bone metabolism. Diabetol Metab Syndr 2017;9:75.

32. Zinman B, Haffner SM, Herman WH, Holman RR, Lachin JM, Kravitz BG, et al. Effect of rosiglitazone, metformin, and glyburide on bone biomarkers in patients with type 2 diabetes. J Clin Endocrinol Metab 2010;95:134-42.

33. Kim MK, Ko SH, Kim BY, Kang ES, Noh J, Kim SK, et al. 2019 Clinical practice guidelines for type 2 diabetes mellitus in Korea. Diabetes Metab J 2019;43:398-406.

34. Buse JB, Wexler DJ, Tsapas A, Rossing P, Mingrone G, Mathieu C, et al. 2019 Update to: management of hyperglycemia in type 2 diabetes, 2018. A consensus report by the American Diabetes Association (ADA) and the European Association for the Study of Diabetes (EASD). Diabetes Care 2020;43:487-93.

35. Matthews DR, Paldanius PM, Proot P, Chiang Y, Stumvoll M, Del Prato S, et al. Glycaemic durability of an early combination therapy with vildagliptin and metformin versus sequential metformin monotherapy in newly diagnosed type 2 diabetes (VERIFY): a 5-year, multicentre, randomised, double-blind trial. Lancet 2019;394:1519-29. 